\title{
Examination of Bunching Effect of Dual-Frequency Modulation in a Traveling Wave Direct Energy Converter Simulator*)
}

\author{
Hiromasa TAKENO, Hiroki SATO, Kazuhiro SHIBATA, Kazuya ICHIMURA \\ and Yousuke NAKASHIMA ${ }^{1)}$ \\ Graduate School of Engineering, Kobe University, Kobe 657-8501, Japan \\ 1) Plasma Research Center, University of Tsukuba, Tsukuba 305-8577, Japan
}

(Received 26 September 2018 / Accepted 30 November 2018)

\begin{abstract}
As for traveling wave direct energy converter (TWDEC), which was expected to be used for energy recovery of fast protons in $\mathrm{D}^{3} \mathrm{He}$ fusion, a new modulation scheme was proposed for better bunching. An analytical formula was derived for complete bunching as an ideal waveform, and use of dual-frequency was proposed for practical application. In the scheme, independent two modulation electrodes were used and applied voltages were obtained by Fourier components of the fundamental and the second harmonic frequencies of the ideal waveform with taking running time between electrodes into account. The effect was confirmed by numerical orbit calculation, and improvement of bunching was found even for the ion beam with energy spread. The effect was indirectly confirmed experimentally by comparing variation of the third harmonic signal with an equivalent value in the orbit calculation. The optimum amplitude of the second harmonic frequency was discussed by comparing the composed waveform with the ideal one.
\end{abstract}

(c) 2019 The Japan Society of Plasma Science and Nuclear Fusion Research

Keywords: advanced fusion, direct energy conversion, TWDEC, modulator, dual-frequency modulation

DOI: $10.1585 /$ pfr.14.2405014

\section{Introduction}

Traveling wave direct energy converter (TWDEC) was proposed as an efficient energy recovery device for fast protons created in $\mathrm{D}-{ }^{3} \mathrm{He}$ fusion [1]. It consists of a modulator and a decelerator. The incident protons are velocity modulated in the modulator, and bunched in the downstream where the decelerator is placed. The bunched ions are trapped in potential valleys of traveling wave in the decelerator, and decelerated with the wave. The bunching is important as it directly relates to conversion efficiency.

The bunching effect depends on a modulation scheme, so the modulation scheme should be studied well. In the previous theoretical and numerical studies, a traveling wave or a standing wave were used [1,2], and 3-stage modulation was also proposed for better modulation effect [3]. In experimental studies, single or a pair of uniform fields were usually used $[4,5]$.

In this paper, a new modulation scheme is proposed for better bunching. An analytical formula of modulation voltage for complete bunching is derived, but it cannot be used in practice as it contains extremely high frequencies. We propose a dual-frequency modulation scheme. In the scheme, independent two modulation electrodes are used and applied voltages are obtained by Fourier components of the fundamental and the second harmonic frequencies of the ideal waveform with taking running time between elec-

author'se-mail: takeno@eedept.kobe-u.ac.jp

*) This article is based on the presentation at the 12th International Conference on Open Magnetic Systems for Plasma Confinement (OS2018). trodes into account. We examine the scheme by numerical orbit calculation. The scheme is also examined by simulation experiment indirectly. From these examinations, we will show the effectiveness of the proposed scheme, and discuss the optimum structure of the dual-frequency modulation.

Our new idea is explained in Sec. 2. Examination by numerical orbit calculation is shown in Sec. 3, that by experiment is in Sec. 4, and discussion is presented in Sec. 5. The contents of the paper are summarized in Sec. 6.

\section{New Modulation Scheme}

\subsection{Waveform of ideal modulation}

We firstly derive the ideal waveform of modulation voltage. Ions with mass of $m$ and velocity of $v_{0}$ are incident in a modulator of uniform field between two grids. The modulation voltage $V_{\mathrm{mi}}(t)$ with period of $T$ is applied to the upstream grid while another one is grounded.

All ions incident in an appropriate period of $t_{0} \leq t \leq$ $t_{0}+T$ have to pass the same position $z_{\mathrm{b}}$ at the same time $t_{\mathrm{b}}$. Thus, necessary velocity of the ion is given by $v(t)=$ $z_{\mathrm{b}} /\left(t_{\mathrm{b}}-t\right)$ when the incident position is taken to be $z=0$. When we introduce $u^{\prime}$ and $u$, which are velocities of the most decelerated and accelerated ions, respectively, $v\left(t_{0}\right)=$ $u^{\prime}$ and $v\left(t_{0}+T\right)=u$ give $t_{\mathrm{b}}=t_{0}+u T /\left(u-u^{\prime}\right)$ and $z_{\mathrm{b}}=$ $u u^{\prime} T /\left(u-u^{\prime}\right)$. Taking normalization of $v / v_{0}=\hat{v}, u / v_{0}=w$, $u^{\prime} / v_{0}=w^{\prime}$, and $\left(t-t_{0}\right) / T=s$, we obtain 


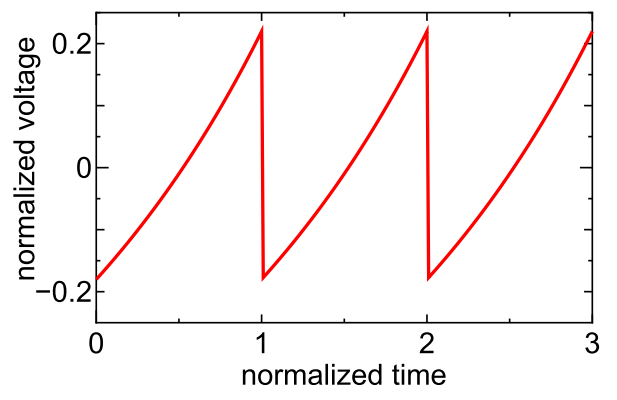

Fig. 1 Ideal waveform of modulation voltage for bunching $\left(U_{\mathrm{m}}=0.2\right)$.

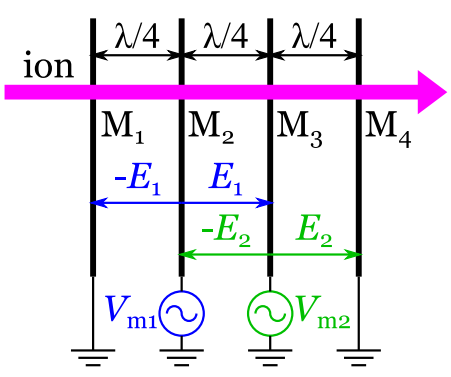

Fig. 2 Practical scheme of dual-frequency modulation.

$$
\hat{v}(s)=\frac{w w^{\prime}}{\left(w^{\prime}-w\right) s+w} .
$$

The energy of modulated ions is $m v(t)^{2} / 2=m v_{0}^{2} / 2+$ $e V_{\mathrm{mi}}(t)$ ( $e$ is unit charge), and $V_{\mathrm{mi}}(t)$ normalized by $m v_{0}^{2} / 2 e$ is $\hat{V}_{\mathrm{mi}}(s)=\hat{v}(s)^{2}-1$. The waveform $\hat{V}_{\mathrm{mi}}(s)$ should not have a dc component, so $\int_{0}^{1} \hat{V}_{\mathrm{mi}}(s) \mathrm{d} s=0$, and thus $w w^{\prime}=1$ is necessary. Finally,

$$
\hat{V}_{\mathrm{mi}}(s)=\frac{1}{\left[\left(w^{\prime}-w\right) s+w\right]^{2}}-1 .
$$

Here, $w$ has a relation of $w^{2}=U_{\mathrm{m}}+\sqrt{U_{\mathrm{m}}^{2}+1}$ where $2 U_{\mathrm{m}}$ gives total amplitude of the normalized waveform. Figure 1 shows a sample waveform of Eq. (2), which quite differs from usual sinusoidal wave.

\subsection{Dual-frequency modulation}

When we use the waveform shown in Fig. 1, we can expect complete bunching, however, it is not practical as the waveform contains extremely high frequency components. We propose approximate usage, that is, modulation composed by two frequencies (dual-frequency modulation). The function of Eq. (2) can be approximated by Fourier series expansion, and we use components of the fundamental and the second harmonic frequencies.

In the dual-frequency modulation, actual structure of the modulator will be as shown in Fig. 2. The distance between electrodes can be appropriate and is taken to be $\lambda / 4$ here, where $\lambda$ is wavelength of traveling wave determined by fundamental modulation frequency and $v_{0}$. When we directly apply two frequency components to electrodes of $\mathrm{M}_{2}$ and $\mathrm{M}_{3}$, we have to consider fields in both front and

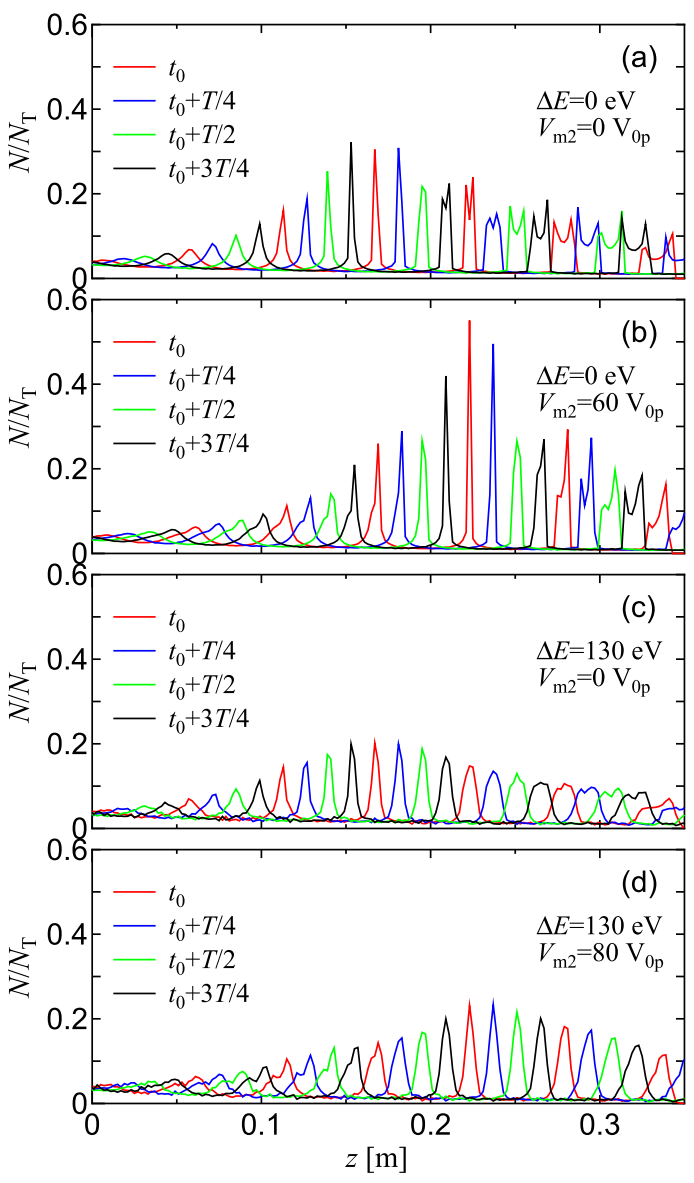

Fig. 3 Axial distribution of ions by orbit calculation.

rear regions for one electrode, and delay of the effect by running time for finite distance between electrodes. As for the latter one, we treat it by a constant delay with assumption of small change of the velocity. In the case of Fig. 2, ion receives fields of $E(t)=-E_{1}(t)+E_{1}(t+T / 4)-$ $E_{2}(t+T / 4)+E_{2}(t+T / 2)$, where $E_{1}(t)=V_{\mathrm{m} 1}(t) /(\lambda / 4)$ and $E_{2}(t)=V_{\mathrm{m} 2}(t) /(\lambda / 4)$. The electrode voltages of $V_{\mathrm{m} 1}$ and $V_{\mathrm{m} 2}$ are determined by correspondence between Fourier components of $E(t)$ and $V_{\mathrm{mi}}(t)$.

\section{Examination by Orbit Calculation}

At first, we performed orbit calculation by using the modulator shown in Fig. 2. Ions are incident sequentially, and time evolution of ion orbit is calculated. Ions change their motion only by the electrode fields and fields by charged particles are not taken into account. Incident energy $E$ has a distribution proportional to exp $[-(E-$ $\left.\left.E_{0}\right)^{2} / 2 \Delta E^{2}\right]$, where $E_{0}(3.2 \mathrm{keV}$ in the present paper) and $\Delta E$ are average energy and energy broadening, respectively. The actual length of $\lambda$ is $0.056 \mathrm{~m}$ in the present condition.

Figure 3 shows axial distribution of ions. The number of ions $N$ are normalized by total number of incident ions for one period of fundamental frequency $\left(N_{\mathrm{T}}=\right.$ 2560). The axial position is described by $z$-axis, the ori- 


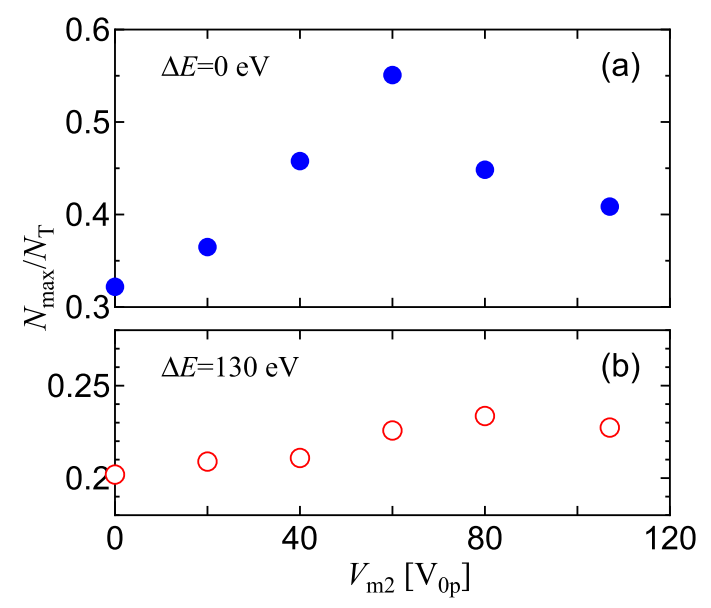

Fig. 4 Variation of the maximum number of ions to amplitude of the second harmonic frequency.

gin of which is taken at the position of $\mathrm{M}_{3}$ in Fig. 2. The curves in red, blue, green and black means snap shots on $t=t_{0}, t_{0}+T / 4, t_{0}+T / 2$, and $t_{0}+3 T / 4$, respectively. We can see the figure as superposition of snap shots, and understand averaged ion distribution in a modulation period.

According to Fig. 3 (a) of $V_{\mathrm{m} 1}=300 \mathrm{~V}_{0 \mathrm{p}}$ and $V_{\mathrm{m} 2}=$ $0 \mathrm{~V}$ for $\Delta E=0 \mathrm{eV}, N / N_{\mathrm{T}}$ is maximum of 0.32 at $z=0.15 \mathrm{~m}$ and this is the bunching point. One one hand, the one is 0.55 at $z=0.22 \mathrm{~m}$ in Fig. 3 (b) when $V_{\mathrm{m} 2}=60 \mathrm{~V}_{0 \mathrm{p}}$. The maximum number of ions $N_{\max }$ increases by application of $V_{\mathrm{m} 2}$, and this is nothing but an improvement of bunching by dual-frequency modulation.

In the actual experiment, we cannot expect $\Delta E=$ $0 \mathrm{eV}$. Figures 3 (c) and (d) show the same comparison for $\Delta E=130 \mathrm{eV}$ which is a typical value in our simulator. On the application of $V_{\mathrm{m} 2}$ (Fig. $\left.3(\mathrm{~d})\right), N / N_{\mathrm{T}}$ is 0.23 , which is superior to that of 0.20 in Fig. 3 (c). Thus, the improvement of bunching can be expected in the simulation experiment.

The variation of $N_{\max }$ was investigated to amplitude of $V_{\mathrm{m} 2}$ when phase of $V_{\mathrm{m} 2}$ relative to $V_{\mathrm{m} 1}$ was fixed to be $0.54 \pi$ obtained by Fourier expansion. The result for $\Delta E=0 \mathrm{eV}$ is shown in Fig. 4 (a). According to the figure, $N_{\max } / N_{\mathrm{T}}$ increases with $V_{\mathrm{m} 2}$, and shows a peak on $V_{\mathrm{m} 2}=60 \mathrm{~V}_{0 \mathrm{p}}$, then it decreases. So, $V_{\mathrm{m} 2}=60 \mathrm{~V}_{0 \mathrm{p}}$ is the most effective. On one hand, in the case of $\Delta E=130 \mathrm{eV}$ in Fig. 4 (b), the variation is small, and $V_{\mathrm{m} 2}=80 \mathrm{~V}_{0 \mathrm{p}}$ is the most effective amplitude. According to the Fourier expansion considered in Sec. 2, corresponding amplitude of $V_{\mathrm{m} 2}$ is $107 \mathrm{~V}_{0 \mathrm{p}}$, which is different from the optimum values shown in Fig. 4.

\section{Examination by Experiment}

\subsection{Experimental equipment}

The dual-frequency modulation was also performed in experiments. Figure 5 shows the experimental simulator of TWDEC. In this experiment, the decelerator was removed and only modulator was installed as in the figure. Helium ions are extracted by extraction voltage of $V_{\mathrm{ex}}=3.2 \mathrm{kV}$

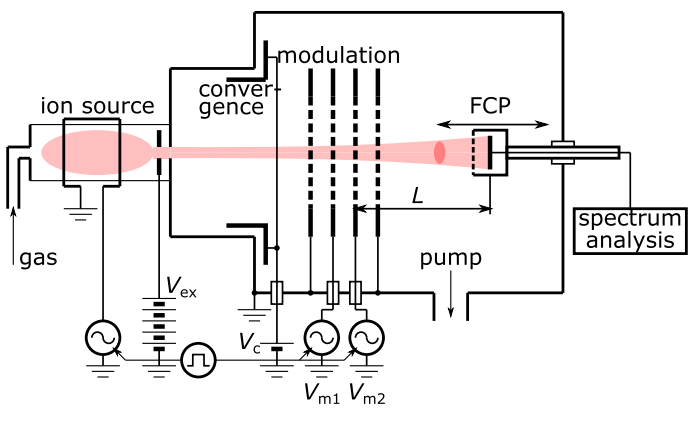

Fig. 5 Schematic illustration of TWDEC simulator.

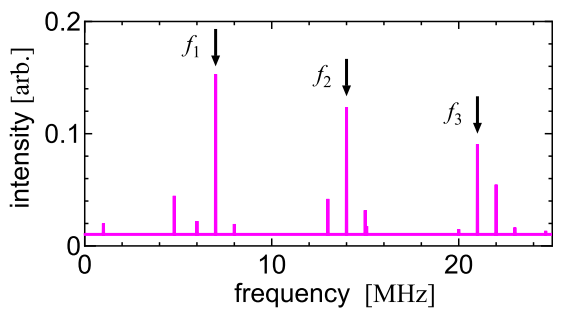

Fig. 6 Sample of measured spectrum of collector current.

from an ion source, which works with repetitive pulse of $1 \mathrm{kHz}$. The extracted beam is converged by convergence voltage of $V_{\mathrm{c}}=530 \mathrm{~V}$. The beam is incident to the modulator, which is designed and composed as explained in Sec. 2. Two RF voltage sources are independent amplifiers, the input signals of which are $7 \mathrm{MHz}$ and $14 \mathrm{MHz}$ and are synchronized. The phase condition is the same as that of orbit calculation. The modulated beam is measured by a Faraday cup (FCP), which is movable in the axial direction. All electrodes in the FCP are not biased, and the signal of the collector current is recorded and its spectrum is analyzed.

\subsection{Experimental results}

A typical example of spectrum-analyzed FCP signal is shown in Fig. 6. We found the third harmonic frequency $f_{3}$ of $21 \mathrm{MHz}$ as well as the fundamental frequency $f_{1}$ of $7 \mathrm{MHz}$ and the second harmonic frequency $f_{2}$ of $14 \mathrm{MHz}$. The axial distribution of ions is a result of non-linear process, so the time variation of FCP signal contains higher harmonic components.

By setting $V_{\mathrm{m} 1}=300 \mathrm{~V}_{0 \mathrm{p}}$ and $V_{\mathrm{m} 2}=80 \mathrm{~V}_{0 \mathrm{p}}$, we measured axial distribution of spectrum intensities of $f_{1}, f_{2}$, and $f_{3}$. The result is shown in Fig. 7 (a), where 3 data were obtained for the same condition. According to the figure, the values for $f_{1}$ and $f_{2}$ vary more widely than those for $f_{3}$ even in the same measurement condition. This is because radiation noise from $\mathrm{M}_{2}$ and $\mathrm{M}_{3}$, to which $V_{\mathrm{m} 1}$ of $f_{1}$ and $V_{\mathrm{m} 2}$ of $f_{2}$ were applied, respectively, affected the measured signals. We concluded the measured values for $f_{1}$ and $f_{2}$ were not reliable. The distribution of the $f_{3}$ component has a peak around $z=0.23-0.24 \mathrm{~m}$.

The dependence on the amplitude of $V_{\mathrm{m} 2}$ was also 

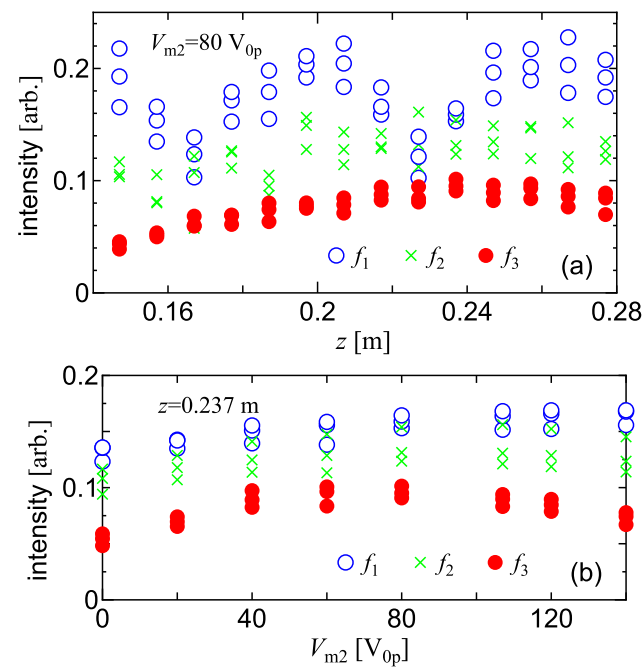

Fig. 7 Variation of measured spectrum intensity.

measured. The result for $z=0.237 \mathrm{~m}$ is shown in Fig. 7 (b). We also find that the values for $f_{1}$ and $f_{2}$ vary more widely than those for $f_{3}$ even in the same measurement condition, and are not reliable. The $f_{3}$ component has a peak around $V_{\mathrm{m} 2}=60-80 \mathrm{~V}_{0 \mathrm{p}}$.

In order to compare between measurement and calculation, the equivalent quantity to experimentally measured data was derived in the orbit calculation in Sec.3. Time evolution of the number of ions in a small spatial element was analyzed by FFT, and components of $f_{1}, f_{2}$, and $f_{3}$ were obtained.

Figure 8 (a) shows comparison of axial distribution of $f_{3}$ between measurement and orbit calculation. Although the ordinates are taken arbitrary, relative variation agrees quite well. The same kind of comparison on $V_{\mathrm{m} 2}$ dependence is also shown in Fig. 8 (b). A good agreement is obtained with the same arbitrary unit as that in Fig. 8 (a).

\section{Discussion}

As explained at the end of Sec. 3, the optimum amplitude of $V_{\mathrm{m} 2}$ is different from that expected by Fourier expansion of the ideal waveform. Figures 9 (a) and (b) show the composed waveform for optimum condition of $V_{\mathrm{m} 2}=60 \mathrm{~V}_{0 \mathrm{p}}$ and Fourier approximation condition of $V_{\mathrm{m} 2}=107 \mathrm{~V}_{0 \mathrm{p}}$, respectively, with the ideal waveform. According to Fig.9(a), agreement of slopes between the composed waveform and the ideal one is better than that in Fig. 9(b). Fourier approximation gives averaged waveform, and agreement of slopes is required for better bunching. This consideration could be confirmed by using another harmonic frequency which has a different slope from the one of the second harmonic frequency.

\section{Summary}

A new modulation scheme was proposed for better bunching in TWDEC. For practical application of the ideal waveform, use of dual-frequency was proposed and in-
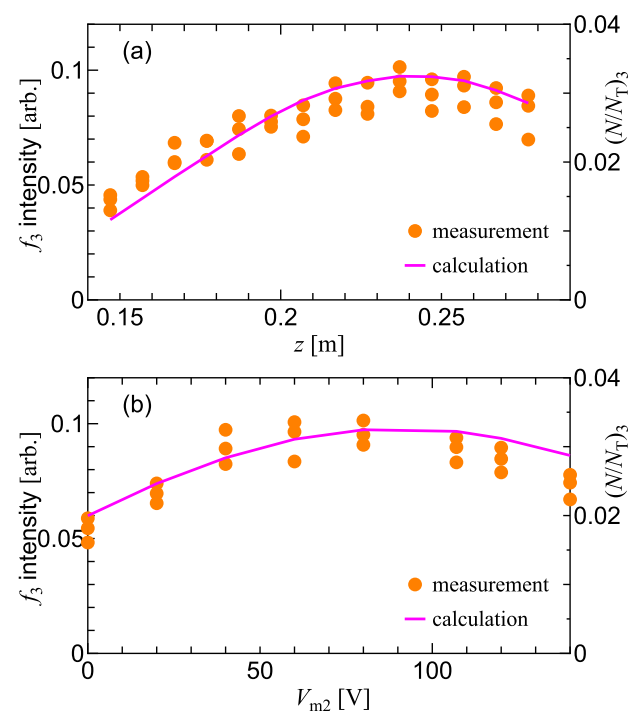

Fig. 8 Comparison between measurement and calculation of spectrum intensity of $f_{3}$.

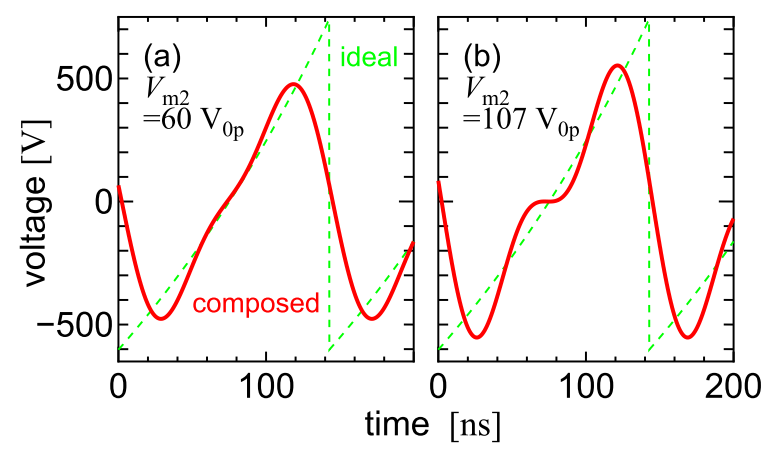

Fig. 9 Variation of composed waveform.

vestigated by numerical orbit calculation and experiment. The better effect was confirmed by numerical orbit calculation, and comparison of the third harmonic components shows good agreement between calculation and measurement. The optimum amplitude of the second harmonic frequency is considered to be determined by agreement of slopes between approximated waveform and ideal one.

\section{Acknowledgments}

The authors acknowledge valuable discussions with Drs. J. Miyazawa and T. Goto. This work was supported in part by a Grant-in-Aid for Scientific Research (16H04317) from Japan Society for the Promotion of Science, and the bilateral coordinated research between Plasma Research Center, University of Tsukuba, National Institute for Fusion Science, and Kobe University (NIFS13KUGM082).

[1] H. Momota, LA-11808-C, Los Alamos Natl. Lab., 8 (1990).

[2] M. Ishikawa et al., Fusion Eng. Des. 41, 541 (1998).

[3] H. Katayama and K. Sato, J. Plasma Fusion Res. 77, 698 (2001) (in Japanese).

[4] H. Takeno et al., Jpn. J. Appl. Phys. 39, 5287 (2000).

[5] Y. Togo et al., Plasma Fusion Res. 10, 3405013 (2015). 